\title{
Evaluación de la competencia motriz en los ámbitos de desarrollo personal y de la productividad de los escolares del municipio de Neiva
}

FERNANDO GALINDO PERDOMO ${ }^{49}$

Programa de Educación Física

Facultad de Educación

fegaper@hotmail.com

\section{Resumen}

E n este documento se dan a conocer los resultados de la evaluación de la Competencia Motriz en escolares del municipio de Neiva, la cual se realizó a partir de la aplicación de una batería de pruebas sobre capacidades físicas coordinativas. La evaluación hizo referencia a las capacidades de combinación y acoplamiento, de diferenciación, de equilibrio estático y dinámico, de reacción motriz y de ritmización. Para cada una de ellas se aplicaron pruebas, las cuales fueron estructuradas por el grupo de investigación Acción Motriz. De cada capacidad coordinativa se establece un referente conceptual con algunos ejemplos para su fácil comprensión. Los resultados obtenidos se muestran en tablas de referencia que constituyen el comportamiento de los escolares evaluados. Estas tablas de referencia servirán a los docentes para que aplique periódicamente las pruebas y compare el resultado de sus escolares con un referente a nivel municipal.

Palabras clave: Capacidades Físicas Coordinativas Combinación y Acoplamiento, Diferenciación, Equilibrio, Reacción, Ritmización, competencia motriz.

\section{Introducción}

La evaluación en la Educación Física es un aspecto que merece mucho cuidado y responsabilidad por parte de los docentes del área. Ésta debe contemplar aspectos motrices, cognitivos y sociales de tal manera que la integración de ellos refleje lo trabajado en las clases.

El presente trabajo propone elaborar tablas de referencia que permitan valorar la competencia motriz a través de pruebas de Capacidades Físicas

${ }^{49}$ Especialista en Pedagogia de la Expresión Lúdica. Docente de Planta de la Universidad Surcolombiana. Integrante del Grupo de investigación ACCION MOTRIZ. Categoria B COLCIENCIAS. 
Coordinativas donde se evalúe la capacidad de combinación y acoplamiento de movimientos, capacidad de diferenciación, çapacidad de equilibrio, capacidad de reacción motriz, y capacidad de producir ritmo o ritmización con el ánimo de estandarizarlas.

Aqui se hace mención sobre los principales resultados que produjo la investigación a través del análisis crítico de los datos de los evaluados, los cuales permitirán establecer un referente sobre el nivel de desempeño que presentan los escolares, con relación a las Capacidades Físicas Coordinativas.

El planteamiento de esta investigación fue elaborado pensando en la necesidad que tiene el profesor de Educación Física de orientar los procesos de formación, de proporcionar elementos conceptuales, metodológicos, didácticos y evaluativos al momento de iniciar un proceso de formación académico con los escolares.

\section{Planteamiento del problema}

¿Cómo evaluar la competencia motriz en los ámbitos de desarrollo personal y de la productividad de los escolares de cinco instituciones del municipio de Neiva?

\section{Diseño metodológico}

Esta investigación se llevó a cabo dentro de los lineamientos de la investigación descriptiva con enfoque cuantitativo. La Unidad de trabajo está constituida por niñas y niños escolares de edades entre 8 y 11 años de 5 instituciones educativas del Municipio de Neiva. La selección de la muestra fue probabilistica.

\section{Objetivo}

Evaluar la competencia motriz en los ámbitos personal y de la productividad de los escolares de cinco instituciones educativas del Municipio de Neiva

\section{Aspectos conceptuales}

\section{Capacidades físicas}

(Murcia, Taborda y Zuluaga, 2004) citando a Muñoz, consideran que en parte las capacidades físicas son "las caracteristicas funcionales de rigor orgánico que cuando son desarrolladas proporcionan al alumno un instrumento sano y un funcionamiento eficiente del mismo, para ser usado cuando pretenda aumentar la destreza de los movimientos de su repertorio motor". En tal sentido estas deben ser entendidas como el resultado de una actividad armónica entre el sistema nervioso central, las diferentes estructuras del sistema muscular y el ambiente social; se deben desarrollar a través de actividades individuales y colectivas teniendo en cuenta caracteristicas biológicas, sociológicas y sicológicas de la persona.

Las capacidades físicas básicas son condiciones internas de cada organismo, que se mejoran por medio de actividades de preparación física, y permiten realizar actividades motrices, ya sean cotidianas o deportivas.

Sobre la clasificación de las capacidades físicas existen diferentes puntos de vista, producto del resultado de estudios realizados por especialistas en el tema; (Ruiz Aguilera, 1996) las agrupa en capacidades condicionales y capacidades coordinativas.

\section{Capacidades físicas coordinativas}

Son particularidades relativamente fijadas y 
generalizadas del desarrollo de los procesos de conducción y regulación de la actividad motora. (Meinel y Schnabel, 2004). (Gallahue. 1982), manifiesta que "la edad sensible para estimular la coordinación, es el periodo en que el organismo presenta las condiciones básicas para desarrollar una capacidad; por esta razón la coordinación no se aprende de un momento a otro sino que se tiene que desarrollar y mejorar a través de métodos técnicos. Por consiguiente, el momento o la etapa en donde se debe estimular, es la fase de Habilidades Motrices Básicas que abarca de los 2 a los 7 años de edad aproximadamente."

Las capacidades coordinativas son determinadas por las funciones parciales que sirven de base para el proceso coordinativo (percepción y elaboración de informaciones, programación y anticipación, comparación de los parámetros ideales y reales del movimiento, impulsos eferentes).

Las capacidades físicas coordinativas según BLUME citado por (López Delgado, 2004) se clasifican en:

Capacidad de Combinación y Acoplamiento: es aquella que permite unir habilidades motrices automatizadas y también se encarga de la coordinación segmentaría de los miembros superiores e inferiores. Se expresa en la interacción de parámetros espaciales, temporales y dinámicos del movimiento. Los métodos para desarrollarla son el encadenamiento gradual, parcial o total entre dos habilidades con acento en algunos componentes; los ejercicios de coordinación segmentaria entre miembros superiores o inferiores, en forma sucesiva, alternada, simultánea con movimientos simétricos, el desarrollo progresivo de la lateralidad, explotando movimientos asimétricos. Algunos ejemplos podrían ser: cualquier recorrido con combinación de habilidades (correr, saltar y girar. Correr saltar y lanzar. Lanzar, aplaudir y tomar el elemento en el aire).

Capacidad de Diferenciación: es la capacidad de lograr una alta exactitud y economía (coordinación fina) de movimiento de cualquier parte del cuerpo y de las fases mecánicas del movimiento total. Permite ejecutar de forma finamente diferenciada los parámetros dinámicos, temporales o espaciales del movimiento sobre la base de percepciones detalladas del tiempo, el espacio y la fuerza.

La capacidad de diferenciación también comprende a la destreza, entendida esta, como la capacidad para llevar a cabo una coordinación fina de movimientos de cabeza, pies y manos; y a la capacidad de distensión muscular (relajación) que conduce a una regulación consciente del tono muscular. Estos son algunos ejemplos: desplazarse en tripedia, juegos de puntería, dribling con ambas manos, alternadas y simultáneas en distintas direcciones.

Capacidad de Equilibrio Estático-Dinámico: (Meinel y Schnabel, 1987) "Es la capacidad de mantener el cuerpo en estado de equilibrio o de recuperarlo después de movimientos o cambios posicionales amplios y veloces. Esta capacidad tiene dos aspectos que deben ser diferenciados". Por un lado, la capacidad de mantener el equilibrio en una posición relativamente estable o en movimientos corporales muy lentos (equilibrio estático); y por otro, la capacidad de mantener o recuperar el estado de equilibrio cuando se realizan cambios importantes y a menudo muy veloces en la posición del cuerpo.

Capacidad de Reacción Motriz: según HIRTZ citado por (Martin, 2001) "se enseña mediante tareas de reacción, con las cuales se aprende a 


\section{Painens Surcolombiara 16}

reaccionar ante señales ópticas, acústicas y ante objetos en movimiento". Las reacciones pueden dividirse en:

Simples: todas las respuestas del organismo, a una señal imprevista ya conocida, en forma de un movimiento claramente definido previamente. Los estimulos pueden ser señales ópticas, acústicas, táctiles o cinestésicas.

Complejas: son todas las respuestas del organismo, en forma de acción motriz no predeterminada, a una señal no claramente conocida. La reacción compleja se basa en gran medida en la capacidad de anticipo y depende notablemente de los conocimientos y la experiencia. Un ejemplo de esta capacidad podría ser: de espaldas o de frente a la dirección de la carrera, según sea conveniente, el ejecutante sale corriendo al escuchar una señal, puede combinarse el estimulo con señales acústicas (silbato), visuales (lanzamiento de una pelota) o táctiles (toque de un compañero).

La Capacidad de Ritmización: capacidad de registrar y reproducir motrizmente un ritmo dado exteriormente y la capacidad de realizar en un movimiento propio el ritmo "interiorizado" por la propia persona, el ritmo de un movimiento existente en la propia imaginación. Forma parte de esta capacidad el saber adaptarse a un ritmo establecido: imprevistamente cambiado. Es determinante en el aprendizaje de cualquier tipo de movimiento y de desarrollo táctico de situaciones en las cuales se proveen variaciones de frecuencia de movimiento. Algunos ejemplos son: desplazamientos con variaciones de ritmo (individual y grupal), responder coordinadamente a ritmos musicales.

(Lagos, 2001) plantea que "es la capacidad de organizar cronológicamente las prestaciones musculares en relación al espacio y al tiempo a partir de un ritmo propuesto externamente o interiorizado por la propia persona"

Para evaluar las capacidades físicas coordinativas de las cuales se hizo referencia al grupo de investigación Acción Motriz, se presentan a continuación los protocolos establecidos para cada una de ellas con sus respectivas imágenes para su fácil comprensión:

\section{Pruebas de capacidades físicas coordinativas}

Capacidad de combinación y acoplamiento del movimiento

Instrucciones: El evaluado se ubica sobre el círculo de $1.50 \mathrm{~m}$. diámetro el cual se encuentra dentro de un círculo concéntrico de $4 \mathrm{~m}$. de diámetro.

El evaluado driblará el balón de baloncesto con su mano hábil, al tiempo que recepciona con su otra mano la pelota de beisbol (lanzamiento) que el evaluador le lanzará de forma parabólica desde fuera del círculo de $4 \mathrm{~m}$ desplazándose alrededor de este. Se realizarán 10 lanzamientos donde se registrarán los puntos obtenidos por el evaluado en la ejecución. Se debe resaltar que los puntos se dan según los errores cometidos en la prueba, los cuales se puntuarán de la siguiente forma:

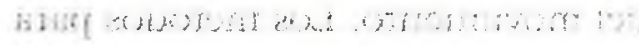

- No coge la pelota y pierde dominio del balón

4 Puntos (4 P)

- Coge la pelota y pierde el dominio del balón

3 Puntos (3 P)

- Apoya la pelota al cuerpo al momento de recepcionarla.

2 Puntos (2 P)

- Dribla fuera del circulo o pisa el exterior del círculo

1 Punto (1 P)

- Coge la pelota sin perder dominio del balón

O Puntos (O P)

Durante el desarrollo de la prueba el evaluador lanzará la pelota aproximadamente cada $5 \mathrm{sg}$. Es importante recomendar que el lanzamiento deba realizarse de tal forma que la pelota llegue al 
evaluado a una altura que se encuentre entre su cresta iliaca y el acromion.

El conteo de los 5 sg por parte del evaluador solo será suspendido cuando el evaluado pierda el control del balón o este salga del círculo de 1.5 $\mathrm{m}$ de diámetro.

La prueba se realizará recepcionando la pelota primero con la mano hábil y luego con la mano contraria, ejecutando 10 recepciones con cada una,

Todos los errores cometidos por los evaluados deben ser registrados, asi estos se presenten de manera simultánea, por ejemplo, dejar caer la pelota y perder el dominio del balón representará una puntuación de 6 , es decir 3 puntos por cada error.

Es necesario aclarar que a menor número de puntos mejor será la valoración obtenida por el evaluado en la prueba.

\section{Material:}

- Un balón de baloncesto No 5, Cinco pelotas de beisbol (lanzamiento), Un círculo de $1.50 \mathrm{~m}$ de diámetro y Un círculo de $4 \mathrm{~m}$ de diámetro.

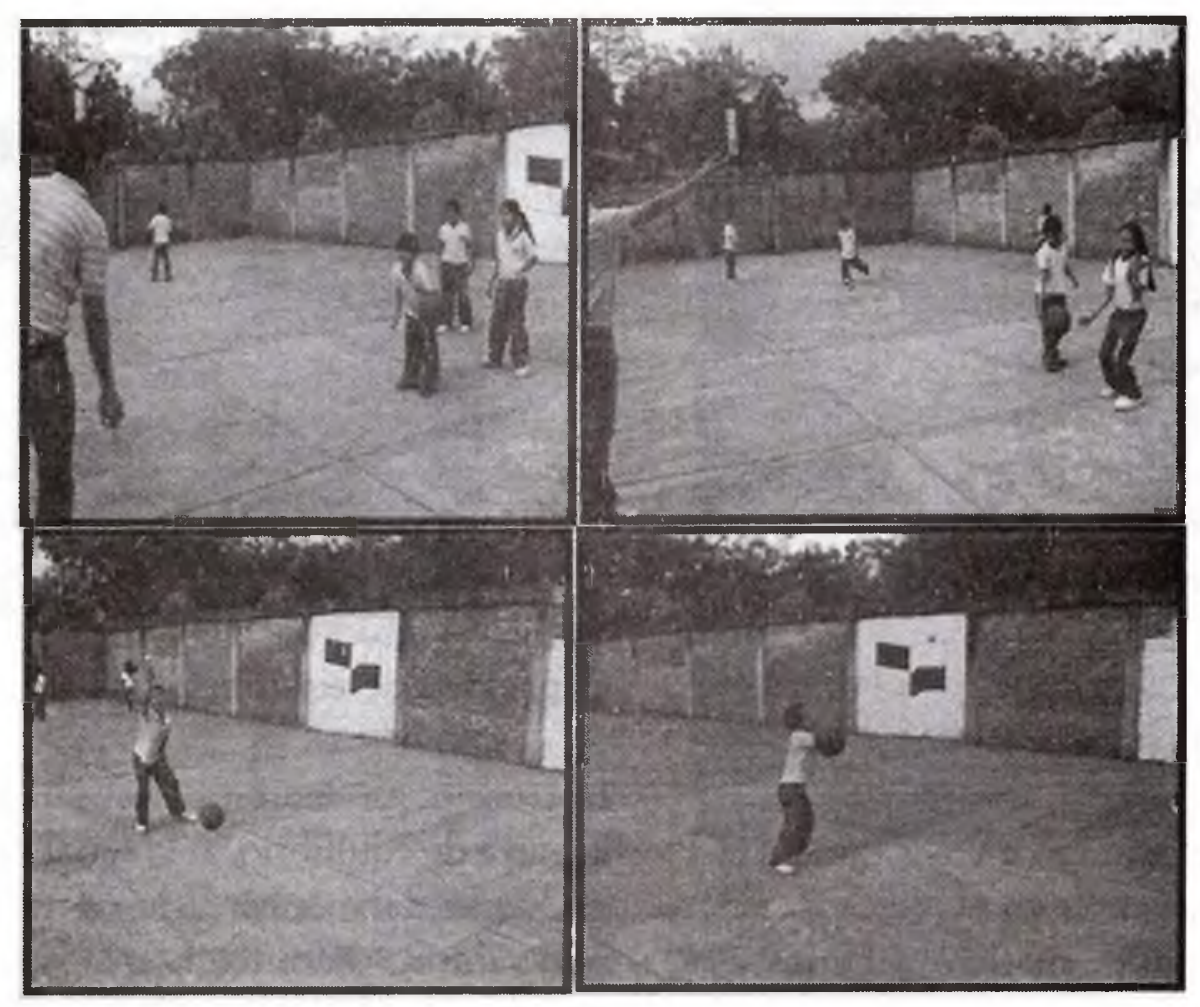

Capacidad de Diferenciación 
Instrucciones: Se ubica un balde en el centro de un círculo, a su alrededor se ubican las 9 pelotas, las cuales se encuentran sobre una linea demarcatoria a las siguientes distancias:

Pelota 1 a $2,5 \mathrm{~m}$.

Pelota 2 a $1,5 \mathrm{~m}$.

Pelota 3 a $3,5 \mathrm{~m}$.

Pelota 4 a $3,5 \mathrm{~m}$.

Pelota 5 a $2,5 \mathrm{~m}$.

Pelota 6 a $1,5 \mathrm{~m}$.

Pelota 7 a $2,5 \mathrm{~m}$.

Pelota 8 a $3,5 \mathrm{~m}$.

Pelota 9 a $1,5 \mathrm{~m}$.

El evaluado se ubica frente de la pelota número 1.

A la señal del evaluador el evaluado debe tomar la primera pelota y lanzarla a la caja tratando de introducirla en ella, luego pasar a la segunda pelota y realizar el mismo ejercicio sucesivamente hasta lanzar todas las pelotas. El evaluado no debe pisar la línea que demarca la distancia de cada lanzamiento. El lanzamiento del evaluado debe realizarse de abajo hacia arriba.

La prueba se evaluará a través de puntos los cuales serán obtenidos de la siguiente manera:

- La pelota no toca el balde

- La pelota golpea el balde pero no entra

- La pelota golpea primero en el balde y después entra en él

- Introduce la pelota de manera 1 Punto (1P) directa en el balde

El objetivo de la prueba es tratar de obtener el mayor número de puntos posibles.

En la ficha de registro se debe colocar la valoración obtenida por el evaluado en cada uno

3 Puntos (3 P)

2 Puntos (2 P) de sus lanzamientos, los cuales serán sumados para determinar su puntuación final.

Material:

- Un balde (ocho litros), 9 pelotas de caucho pequeñas y Cinta métrica

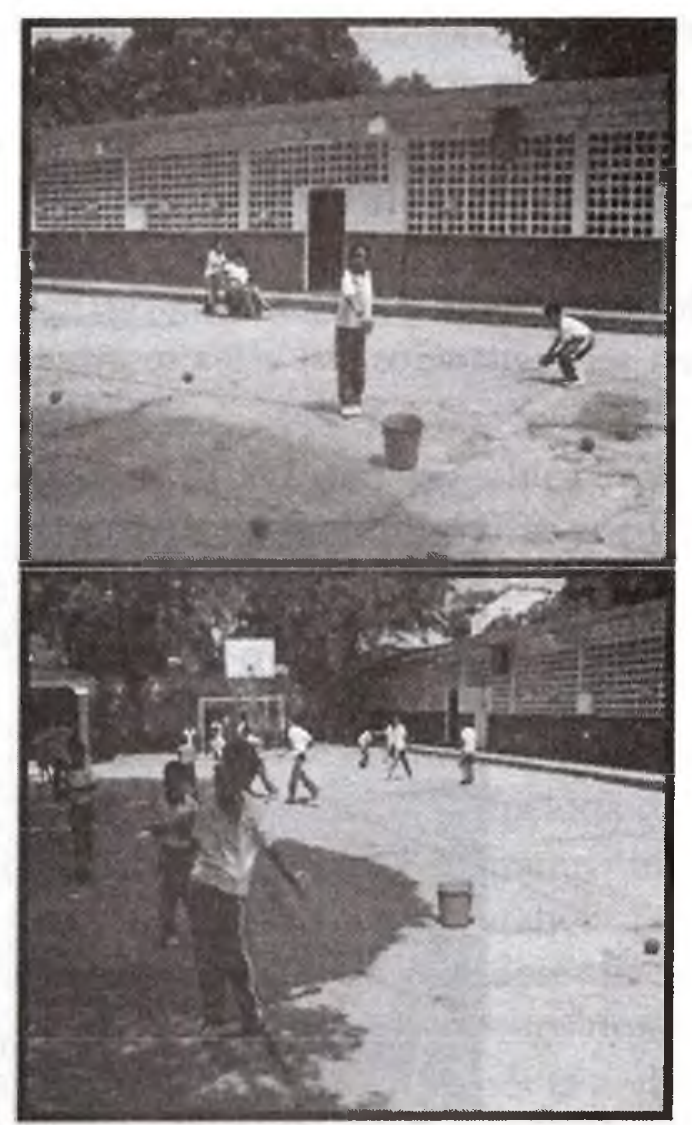

4 Puntos (4 P) Capacidad de equilibrio

Instrucciones: Se ubica al evaluado al frente de la barra de equilibrio. A la señal del evaluador el evaluado debe subir a la barra de equilibrio realizar una detención de 5 sg, sobre el pie derecho, desplazarse por la barra hasta el primer metro donde realizará una segunda detención de 5 sg con el mismo pie, estas detenciones se realizarán también a los $2 \mathrm{~m}$ de distancia y al final de la barra de equilibrio. El evaluado al finalizar el recorrido deberá bajar de la barra y posteriormente volver a subir a ella, para realizar 


\section{Panden Suncolombiana 16}

un recorrido igual al ejecutado anteriormente, pero en cada detención deberá apoyar el pie izquierdo. La prueba se evaluará a través de puntos los cuales serán obtenidos de la siguiente manera:

- Cae de la barra

3 Puntos (3 P)

- Coloca sus dos pies sobre

2 Puntos (2 P) la barra

- Junta su pie libre con el pie

1 Punto (1 P) de apoyo

- Realiza el ejercicio

1 Punto (1 P) correctamente

La valoración de los evaluados será inversamente proporcional a los puntos obtenidos por cada individuo, es decir que a menor número de puntos mejor será la valoración obtenida y viceversa.

Cada uno de los errores cometidos por el evaluado serán tomados en forma independiente, en caso de presentarse situaciones simultaneas como por ejemplo que el evaluado en una detención coloque los dos pies sobre la barra y casi de inmediato caiga de la misma; en esta situación el error a tener en cuenta será el que represente un mayor valor en puntos, para nuestro ejemplo seria igual a 3 puntos (caer de la barra).

Material:

- Una barra de equilibrio construida por el docente o adquirida por la institución educativa de $3 \mathrm{~m}$ de largo por $35 \mathrm{~cm}$. de alto y $10 \mathrm{~cm}$. de ancho (según modelo) y Un cronometro.

\section{Capacidad de reacción}

Instrucciones: Se sienta al evaluado de frente a un metro atrás de los primeros dos conos que

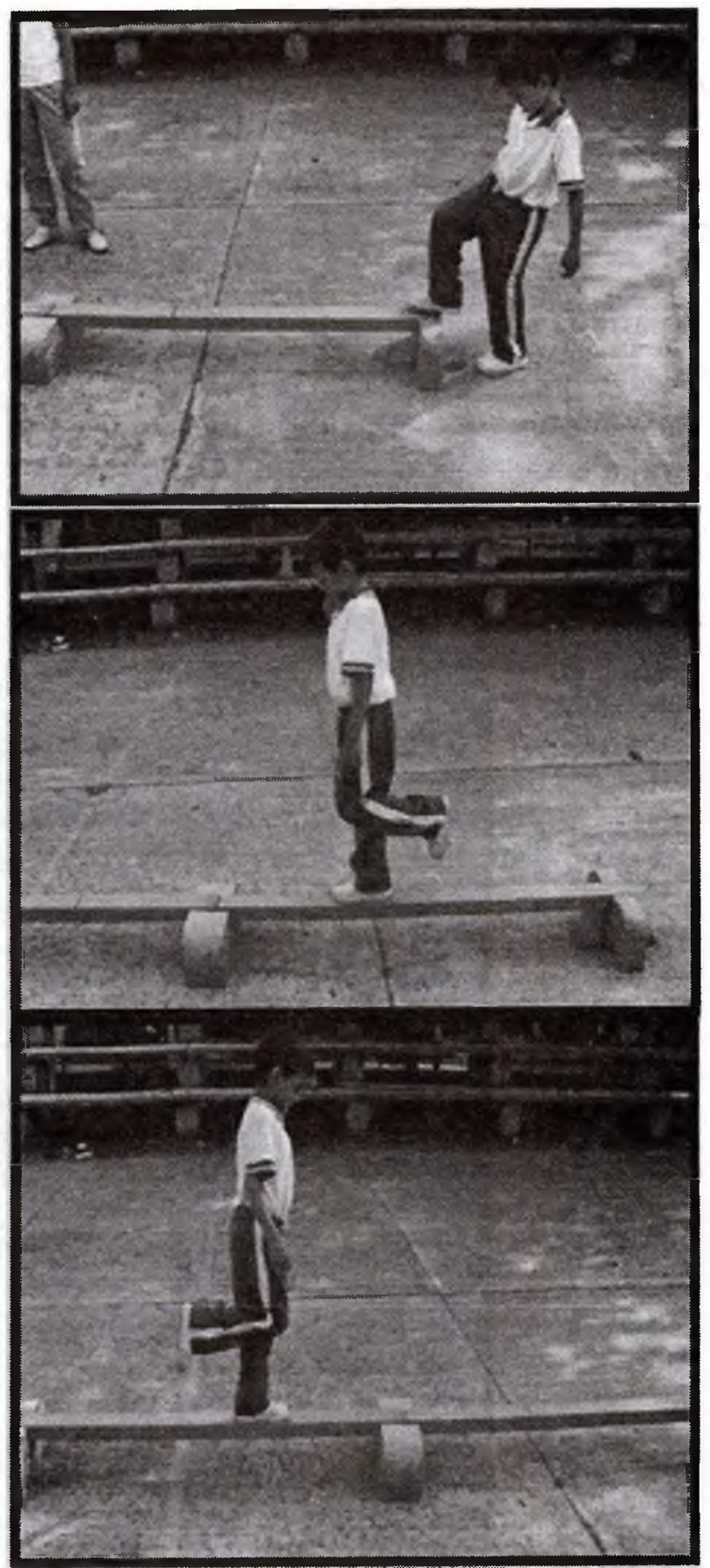

están separados 5 metros entre sí. Frente a él se abrirá un espacio de $6 \mathrm{~m}$ el cual presentará líneas demarcatorias a los 2,4 y $6 \mathrm{~m}$. de distancia respectivamente.

El evaluador pondrá una pelota sobre una de las rampas para que esta baje y ruede hacia el evaluado, quien al momento de ver la pelota, sin necesidad de mirar atrás, debe levantarse y correr 
a atraparla lo antes posible. Se efectuarán 6 lanzamientos a lados distintos del evaluado.

El test se evaluará a través de la obtención de puntos, los cuales serán asignados a los evaluados según los siguientes parámetros:

- No atrapa la pelota

- Atrapa la pelota entre los 4 y 6 metros

- Atrapa la pelota entre los 2 y 4 metros

- Atrapa la pelota antes de los 2 metros

3 Puntos (3 P)

2 Puntos (2 P)

1 Punto (1 P)
Las rampas de lanzamiento deben de ubicarse con un ángulo de $45^{\circ}$ para permitir que la pelota logre una velocidad apropiada y constante y para evitar que al momento de golpear el terreno produzca sonidos que puedan advertir al evaluado sobre la dirección de la misma.

\section{Material:}

- Dos pelotas de beisbol, Dos rampas para lanzamiento de $1.50 \mathrm{~m}$ (tuvo P.V.C de 3 pulgadas), Cinta métrica y ocho conos.

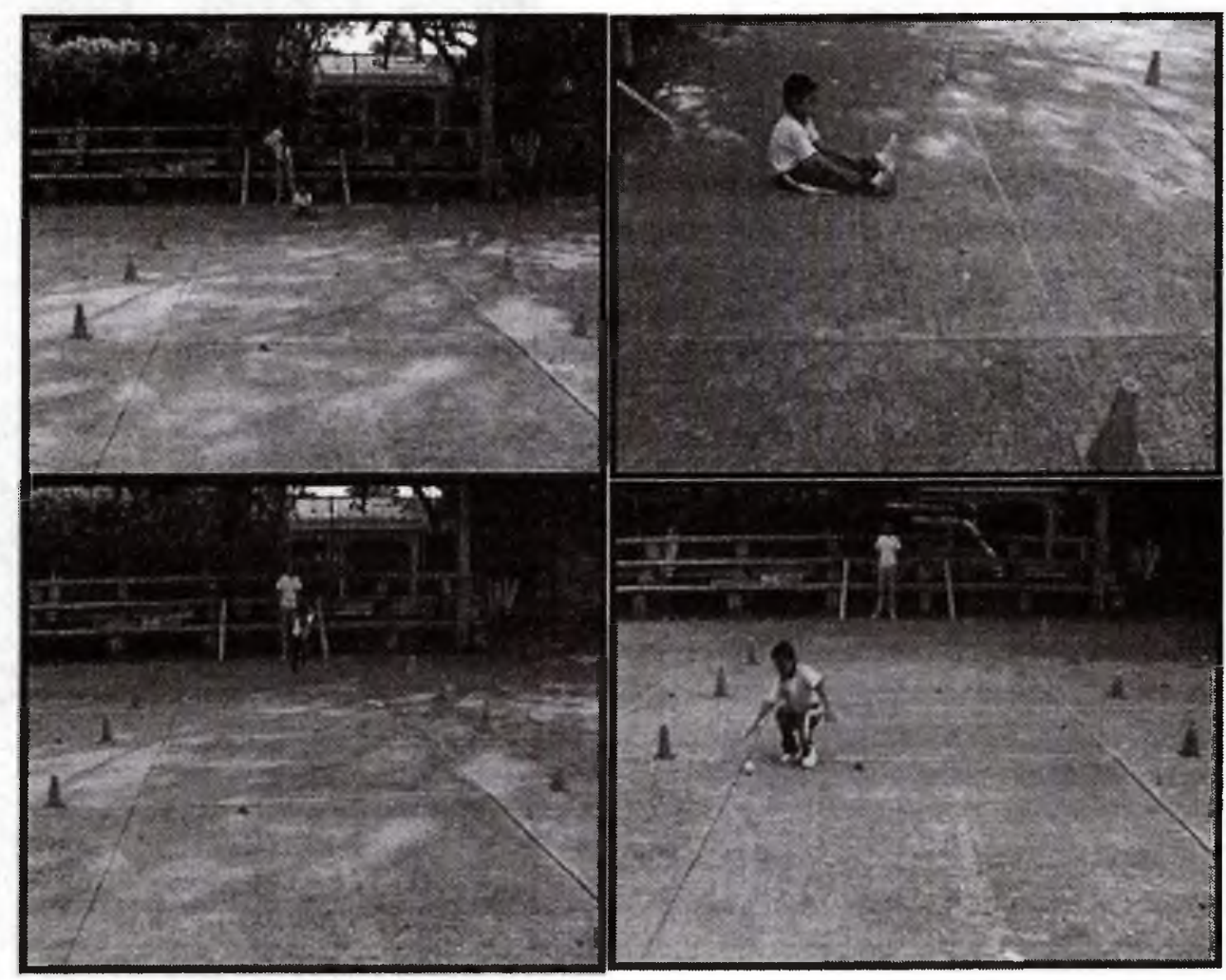

\section{Capacidad de ritmización}

Instrucciones: se ubica al niño en el punto de inicio con su respectiva soga. A la señal dada por el evaluador el evaluado iniciará a saltar la soga efectuando la siguiente secuencia: 5 saltos a pie junto, 5 saltos con pie derecho, 5 saltos con pie izquierdo y 5 saltos a pie junto. Se evaluará a través de puntos, asignando a cada error cometido un total de 2 puntos. Asi la sumatoria de los puntos obtenidos según los errores representarán la valoración del individuo. En cada ocasión que el evaluado cometa un error deberá continuar desde el punto en el que se encontraba. La cantidad de puntos obtenidos en la prueba serán inversamente proporcional a la valoración asignada al individuo, es decir que a mayor número de puntos obtenidos en la prueba, menor valoración en esta capacidad. 
Material:

- Una soga adaptada a la estatura del evaluado.

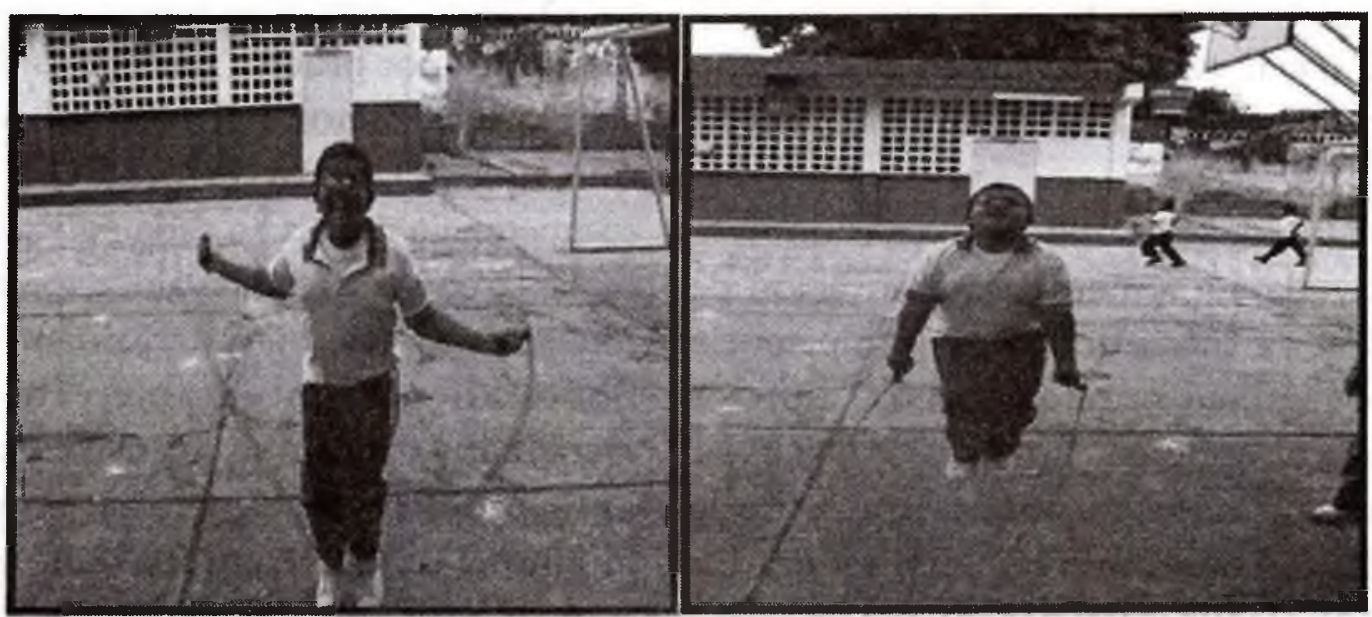

\section{Resultados e interpretación de los datos}

Después de haber obtenido los resultados de las diferentes instituciones, procedimos a ingresar los datos al programa SPSS donde se ubicaron cada una de las variables establecidas en la ficha de recolección de datos y con ella pudimos analizar algunas medidas estadísticas como la media, la mediana, el dato mínimo, el máximo y la desviación estándar; esto nos ayudó a realizar un análisis de los datos obtenidos por prueba, por grupo de edad, por institución y desarrollar comparaciones teniendo en cuenta las diferentes variables objeto de estudio en las instituciones trabajadas.
Una vez hecho este paso, procedimos a ubicar los datos en el programa EXCEL donde elaboramos dos tablas, una con los datos de las mujeres y otra con los datos de los hombres; con los datos de mínimo y máximo pudimos hallar la diferencia entre los dos datos anteriores; al tener la diferencia, esta se dividió en cinco (5) teniendo en cuenta las valoraciones cuantitativas que se utilizan normalmente en las instituciones para evaluar los procesos académicos. Para facilitar su comprensión se realizó una equivalencia entre la valoración cuantitativa actual con la cualitativa que estaba establecida anteriormente.

Tabla 1. Equivalencia valoración cualitativa y la cuantitativa

\begin{tabular}{|c|c|}
\hline VALORACION CUALITATIVA & VALORACION CUANTITATIVA \\
\hline E & Estudiantes que obtienen entre 4.6 y 5.0 \\
\hline S & Estudiantes que obtienen entre 4.0 a 4.5 \\
\hline A & Estudiantes que obtienen entre 3.0 y 3.99 \\
\hline I & Estudiantes que obtienen entre 2 y 2.99 \\
\hline D & Nota inferior a 2.0 \\
\hline
\end{tabular}


Este paso nos ayudó para crear las tablas con los datos establecidos para cada valoración, de tal forma que el docente pueda aplicarlas en su institución y teniendo en cuenta él resultado pueda evaluar al educando en su desempeño motriz.

Estas tablas son de gran utilidad para el docente porque le ayudará a fomentar la motivación de los estudiantes a través de la sana competencia entre ellos; también se reforzarán los aspectos cognitivos inculcando en ellos el valor de cuidar su cuerpo y su salud corporal a través de la actividad física constante. También tendrá un referente a nivel municipal para saber cómo está el educando de esa institución con respecto a los de otras instituciones y también podrá tener un consolidado de toda la institución que le facilitará la selección de los estudiantes que representaran su colegio en los diferentes deportes en juegos intercolegiados.

Para facilitar los procesos de evaluación y teniendo en cuanta las condiciones fisiológicas y motrices de los niños hemos dividido los resultados por grupos de edad quedando establecidos de la siguiente manera: primer grupo 8 y 9 años, segundo grupo 10 y 11 años.
Tablas para valorar la competencia motriz en el ámbito personal y de la productividad

Después de haber hecho el análisis de los resultados por prueba, por grupo de edad y por género, nos dimos a la tarea de realizar tablas que nos permitan valorar el estado de la competencia motriz en cada educando. En estas tablas se encuentran las valoraciones cualitativas que se establecieron anteriormente (Excelente, Sobresaliente, Aceptable, Insuficiente y Deficiente), para que los estudiantes una vez aplicados los test puedan saber cómo se encuentran en comparación con sus compañeros; a demás, el docente podrá valorar el desempeño de sus escolares y con los resultados organizar planes de mejoramiento. Hay que aclarar que esta valoración no será la nota definitiva del periodo, hay que acogerla como una evaluación formativa, debido que faltaria evaluar el aspecto cognitivo y el social que son factores indispensables en la formación integral de los educandos. Estas tablas igualmente, sirven para que los docentes que orientan el área de Educación física puedan conocer una vez obtenidos los resultados cómo está su institución con respecto con un referente municipal.

Grupo de edad 8 y 9 años

Grupo de edad 8 y 9 años

Tabla 2. Tabla de valoración prueba combinación y acoplamiento mano diestra

\begin{tabular}{|l|c|c|c|c|c|}
\hline \multicolumn{7}{|c|}{ TEST DE COMB. ACOPL ( M. DIESTRA) TOTAL DE PUNTOS } \\
\hline \multicolumn{5}{|c|}{ VALORACIÓN } \\
\hline GENERO & DEFICIENTE & INSUFICIENTE & ACEPTABLE & SOBRESALIENTE & EXCELENTE \\
\hline FEMENINO & 17 Y MÁS & 13 A 16 & 8 A 12 & 4 A 7 & 3 O MENOS \\
\hline MASCULINO & 17 Y MÁS & 13 A 16 & 10 A 12 & 6 A 9 & 5 O MENOS \\
\hline
\end{tabular}


Tabla 3. Tabla de valoración prueba combinación y acoplamiento mano contraria

\begin{tabular}{|l|c|c|c|c|c|}
\hline \multicolumn{5}{|c|}{ PALUEBA DE ACOPLAMIENTO ( M.CONTRARIA) TOTAL DE PUNTOS } \\
\hline \multicolumn{5}{|c|}{ VALIÓN } \\
\hline GENERO & DEFICIENTE & INSUFICIENTE & ACEPTABLE & SOBRESALIENTE & EXCELENTE \\
\hline FEMENINO & 17 Y MÁS & 13 A 16 & 8 A 12 & 4 A 7 & 3 O MENOS \\
\hline MASCULINO & 18 Y MÁS & 14 A 17 & 10 A 13 & 6 A 9 & 5 O MENOS \\
\hline
\end{tabular}

Tabla 4. Tabla de valoración prueba de reacción

\begin{tabular}{|l|c|c|c|c|c|}
\hline \multicolumn{7}{|c|}{ PRUEBA DE REACCION - TOTAL DE PUNTOS } \\
\hline \multicolumn{5}{|c|}{ VALORACIÓN } \\
\hline GENERO & DEFICIENTE & INSUFICIENTE & ACEPTABLE & SOBRESALIENTE & EXCELENTE \\
\hline FEMENINO & 15 Y MÁS & 12 A 14 & 10 A 11 & 7 A 9 & 6 O MENOS \\
\hline MASCULINO & 15 Y MAS & 13 A 14 & 12 A 12 & 10 A 11 & 9 O MENOS \\
\hline
\end{tabular}

Tabla 5. Tabla de valoración prueba de diferenciación

PRUEBA DE DIFERENCIACION - TOTAL DE PUNTOS

\section{VALORACIÓN}

\begin{tabular}{|l|c|c|c|c|c|}
\hline GENERO & DEFICIENTE & INSUFICIENTE & ACEPTABLE & SOBRESALIENTE & EXCELENTE \\
\hline FEMENINO & 20 Y MÁS & 17 A 19 & 13 A 16 & 10 A 12 & 9 O MENOS \\
\hline MASCULINO & 20 Y MÁS & 15 A 19 & 10 A 14 & 5 A 9 & 4 O MENOS \\
\hline
\end{tabular}


Tabla 6. Tabla de valoración prueba de equilibrio

\begin{tabular}{|l|l|c|c|c|c|}
\hline \multicolumn{5}{|c|}{ PRUEBA DE EQUILIBRIO - TOTAL DE PUNTOS } \\
\hline \multicolumn{5}{|c|}{ VALORACIÓN } \\
\hline GENERO & DEFICIENTE & INSUFICIENTE & ACEPTABLE & SOBRESALIENTE & EXCELENTE \\
\hline FEMENINO & 6 Y MÁS & 5 A 5 & 3 A 4 & 2 A 2 & 1 M MENOS \\
\hline MASCULINO & 10 Y MAS & 7 A 9 & 5 A 6 & 2 A 4 & 1 MENOS \\
\hline
\end{tabular}

Tabla 7. Tabla de valoración prueba de ritmización

\begin{tabular}{|l|c|c|c|c|c|}
\hline \multicolumn{7}{|c|}{ PRUEBA DE RITMIZACION - TOTAL DE PUNTOS } \\
\hline \multicolumn{5}{|c|}{ VALORACIÓN } \\
\hline GENERO & DEFICIENTE & INSUFICIENTE & ACEPTABLE & SOBRESALIENTE & EXCELENTE \\
\hline FEMENINO & 32 Y MÁS & 24 A 31 & 16 A 23 & 8 A 15 & 7 o MENOS \\
\hline MASCULINO & 32 Y MÁS & 24 A 31 & 16 A 23 & 8 A 15 & 7 o MENOS \\
\hline
\end{tabular}

Grupo de edad 10 y 11 años

Tabla 8. Tabla de valoración prueba combinación y acoplamiento mano diestra

\begin{tabular}{|c|c|c|c|c|c|}
\hline \multicolumn{7}{|c|}{ TEST DE COMB. ACOPL ( M. DIESTRA) TOTAL DE ERRORES } \\
\hline SEXO & DEFICIENTE & INSUFICIENTE & ACEPTABLE & SOBRESALIENTE & EXCELENTE \\
\hline MUJERES & 17 Y MÁS & 13 A 16 & 10 A 12 & 6 A 9 & 5 O MENOS \\
\hline HOMBRES & 16 Y MÁS & 12 A 15 & 9 A 11 & 5 A 8 & 4 O MENOS \\
\hline
\end{tabular}


Tabla 9. Tabla de valoración prueba combinación y acoplamiento mano contraria

PRUEBA DE COMB. ACOPLAMIENTO ( M.CONTRARIA) TOTAL DE ERRORES

\section{VALORACIÓN}

\begin{tabular}{|l|c|c|c|c|c|}
\hline SEXO & DEFICIENTE & INSUFICIENTE & ACEPTABLE & SOBRESALIENTE & EXCELENTE \\
\hline MUJERES & 16 Y MÁS & $12 \mathrm{~A} 15$ & $8 \mathrm{~A} 11$ & $4 \mathrm{~A} 7$ & 3 O MENOS \\
\hline HOMBRES & $16 \mathrm{Y}$ MÁS & $12 \mathrm{~A} 15$ & $8 \mathrm{~A} 11$ & $4 \mathrm{~A} 7$ & 3 O MENOS \\
\hline
\end{tabular}

Tabla 10. Tabla de valoración prueba de reacción

PRUEBA DE REACCION -TOTAL DE PUNTOS

\section{VALORACIÓN}

\begin{tabular}{|l|c|c|c|c|c|}
\hline GENERO & DEFICIENTE & INSUFICIENTE & ACEPTABLE & SOBRESALIENTE & EXCELENTE \\
\hline FEMENINO & 15 Y MÁS & $13 \mathrm{~A} 14$ & $10 \mathrm{~A} 12$ & $8 \mathrm{~A} 9$ & 7 O MENOS \\
\hline MASCULINO & 14 Y MÁS & $12 \mathrm{~A} 13$ & $10 \mathrm{~A} 11$ & $8 \mathrm{~A} 9$ & 7 O MENOS \\
\hline
\end{tabular}

Tabla 11. Tabla de valoración prueba de diferenciación

PRUEBA DE DIFERENCIACION- TOTAL DE ERRORES

\section{VALORACIÓN}

\begin{tabular}{|l|c|c|c|c|c|}
\hline SEXO & DEFICIENTE & INSUFICIENTE & ACEPTABLE & SOBRESALIENTE & EXCELENTE \\
\hline MUJERES & 21 Y MÁS & 17 A 20 & 12 A 16 & 8 A 11 & 7 O MENOS \\
\hline HOMBRES & 16 Y MÁS & 13 A 15 & 9 A 12 & 6 A 8 & 5 O MENOS \\
\hline
\end{tabular}


Tabla 12. Tabla de valoración prueba de equilibrio

\begin{tabular}{|c|c|c|c|c|c|}
\hline \multicolumn{6}{|c|}{ PRUEBA DE EQUILIBRIO - TOTAL DE ERRORES } \\
\hline \multicolumn{6}{|c|}{ VALORACIÓN } \\
\hline SEXO & $\begin{array}{c}\text { DEFICIEN } \\
\text { TE }\end{array}$ & $\begin{array}{c}\text { INSUFICIEN } \\
\text { TE }\end{array}$ & $\begin{array}{c}\text { ACEPTAB } \\
\text { LE }\end{array}$ & $\begin{array}{c}\text { SOBRESALIE } \\
\text { NTE }\end{array}$ & $\begin{array}{l}\text { EXCELEN } \\
\text { TE }\end{array}$ \\
\hline $\begin{array}{l}\text { MUJER } \\
\text { ES }\end{array}$ & 5 Y MÁS & $4 \mathrm{~A} 4$ & 2 A 3 & $1 \mathrm{~A} 1$ & 0 \\
\hline $\begin{array}{l}\text { HOMBR } \\
\text { ES }\end{array}$ & 2 Y MAS & 2 A 2 & $1 \mathrm{~A} 2$ & $1 \mathrm{~A} 1$ & 0 \\
\hline
\end{tabular}

Tabla 13. Tabla de valoración prueba de ritmización

PRUEBA DE RITMIZACION - TOTAL DE ERRORES

\begin{tabular}{|l|c|c|c|c|c|}
\hline \multicolumn{6}{|c|}{ VALORACIÓN } \\
\hline SEXO & DEFICIENTE & INSUFICIENTE & ACEPTABLE & SOBRESALIENTE & EXCELENTE \\
\hline MUJERES & 19 Y MAS & $14 \mathrm{~A} 18$ & $10 \mathrm{~A} 13$ & $5 \mathrm{~A} 9$ & 4 o MENOS \\
& & & & $8 \mathrm{~A} 15$ & 7 MENOS \\
\hline HOMBRES & 32 Y MAS & $24 \mathrm{~A} 31$ & $16 \mathrm{~A} 23$ & & \\
\hline
\end{tabular}

\section{Recomendaciones}

Una vez vivenciada la aplicación de las pruebas en cada una de las instituciones educativas seleccionadas como muestra, realizado el análisis de los resultados y haber elaborado las tablas de valoración, es importante dar a conocer algunas recomendaciones que ayudarán para que los procesos de formación integral de los escolares en el nivel de primaria del municipio de Neiva mejoren a través de un trabajo ordenado y secuencial. Igualmente estas recomendaciones ayudarán a que los estudiantes, docentes del área y personal encargado de orientar esta asignatura ayuden a incrementar los resultados obtenidos, además se concienticen de la importancia de realizar actividad física constante para el bienestar personal en aspectos como salud e higiene, de 
tal manera que mejorando estos aspectos mejorará también su competencia motriz.

- Es indispensable la presencia de un docente de Educación Física titulado en el área, quien sea el encargado de orientar los procesos de formación de los educandos en este nivel educativo. Si esto se pudiera lograr, los escolares del nivel de primaria no tendrian las falencias que presentan al llegar a secundaria.

- Es indispensable que los docentes que orientan actualmente el área de Educación Física, organicen el trabajo con los estudiantes de su curso, de tal manera que se trabajen las capacidades físicas coordinativas a través de actividades recreativas y deportivas.

- Concientizar a los escolares del municipio de Neiva de básica primaria, para que realicen actividad física de forma constante no solo en la clase de Educación Física, sino también, en el tiempo libre. Si este aspecto se pudiera lograr los resultados de las pruebas mejorarían notablemente en un tiempo muy corto.

- Que las instituciones educativas adquieran y faciliten material didáctico y deportivo a las distintas sedes, para que los docentes encargados de orientar las clases de Educación Física puedan utilizar el material básico necesario para desarrollar un tema específico; de esta manera se evitaría que las clases continuaran siendo solamente dar un balón a los niños para que hagan lo que ellos quieran.

- Que los docentes encargados de estructurar el plan de estudios del área, incluyan dentro de la programación de cada uno de los grados la aplicación periódica de las pruebas para tener un control permanente sobre el trabajo realizado con los escolares. Los resultados obtenidos cada vez que aplicamos las pruebas, nos darán un referente sobre el avance o no de los escolares con respecto a la competencia motriz.

- Los estudiantes con buena competencia motriz, serán emocionalmente estables, se les facilitará la asimilación de fundamentos deportivos y mejorará la productividad en la escuela y en la vida cotidiana.

\section{Bibliografía}

CAMACHO COY, Hipólito. CASTILLO LUGO, Eduardo. MONJE MAHECHA, Jaime. RAMIREZ LOPEZ, Gloria Milena. Competencias y estándares para El área de Educación Física "una experiencia que se construye paso a paso". Ed. Kinesis. Colombia. Primera edición 2008.

GALLAHUE, David. Capacidades Coordinativas. Pagina web. www.efdeportes.com/Revista Digital. 2001.

GROSSER, manfred y NEUMAIER, August, Teoria y practica de los test deportivos-motores. Editorial Paidotribo. 1988 p.20

LAGO PEÑAs, Carlos. GRAÑA LOPEZ, Pilar. www.efdeportes.com . Las capacidades coordinativas en los juegos colectivos deportivos. El balonmano. 2001.

LOPEZ DELGADO, Eduardo. Las Capacidades Motrices. Conferencia Internacional de entrenamiento deportivo. La habana (cuba). 2004.

MARTIN, Dietrich; CARL, Klaus; LEHNERTZ, Klaus. Manual de metodología del entrenamiento deportivo. Ed. Paidotribo. Barcelona. 2001.

MEINEL, Kurt; SCHNABEL, Gunter. Teoría del movimiento. Motricidad deportiva. Ed. Stadium. Buenos Aires. 2004.

MURCIA, Napoleón; TABORDA, Javier; ZULUAGA, Luis Fernando. Escuelas de formación deportiva y entrenamiento deportivo infantil. Ed. Kinesis. Armenia. 2004

RUIZ AGUilerA, Ariel. El desarrollo y perfeccionamiento de las capacidades físicas. Tomado del colectivo de autores cubanos. En: desarrollo de capacidades fisico motrices. 1996. 